\title{
Systematic identification of key functional modules and genes in esophageal cancer
}

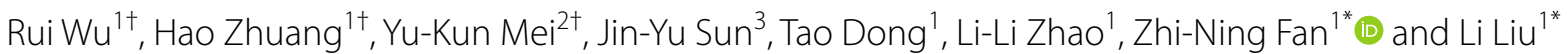

\begin{abstract}
Background: Esophageal cancer is associated with high incidence and mortality worldwide. Differential expression genes (DEGs) and weighted gene co-expression network analysis (WGCNA) are important methods to screen the core genes as bioinformatics methods.

Methods: The DEGs and WGCNA were combined to screen the hub genes, and pathway enrichment analyses were performed on the hub module in the WGCNA. The CCNB1 was identified as the hub gene based on the intersection between DEGs and the greenyellow module in WGCNA. Expression levels and prognostic values of CCNB1 were verified in UALCAN, GEPIA2, HCMDB, Kaplan-Meier plotter, and TIMER databases.
\end{abstract}

Results: We identified 1,044 DEGs from dataset GSE20347, 1,904 from GSE29001, and 2,722 from GSE111044, and 32 modules were revealed by WGCNA. The greenyellow module was identified as the hub module in the WGCNA. CCNB1 gene was identified as the hub gene, which was upregulated in tumour tissues. Moreover, esophageal cancer patients with higher expression of CCNB1 showed a worse prognosis. However, CCNB1 'might not play an important role in immune cell infiltration.

Conclusions: Based on DEGs and key modules related to esophageal cancer, CCNB1 was identified as the hub gene, which offered novel insights into the development and treatment of esophageal cancer.

Keywords: Esophageal cancer, Integrated transcriptomic analysis, Weighted gene co-expression network analysis, Bioinformatics, CCNB1

\section{Introduction}

Esophageal cancer (ESCA) is the seventh most common cancer and the sixth leading cause of cancer-related death worldwide [1, 2]. In 2018, there were 572,034 new victims and 508,585 deaths globally, and almost half of the cases occur in China $[1,3]$. The 5-year survival rate of earlystage cancer may reach $85 \%$, while it drops to less than $15 \%$ when the cancer progress to advanced stage $[4,5]$. Despite the rapid development of multimodal treatments in recent years, esophagectomy remained the standard

\footnotetext{
*Correspondence: fanzhining@njmu.edu.cn; Kit9178@sina.com

${ }^{\dagger}$ Rui Wu, Hao Zhuang and Yu-Kun Mei contributed equally to this work ${ }^{1}$ Department of Digestive Endoscopy, The First Affiliated Hospital with Nanjing Medical University, 300 Guangzhou Road, Nanjing 210029, Jiangsu, China

Full list of author information is available at the end of the article
}

curative method for advanced ESCA, and patients still experience poor life quality and face risk of long-term recurrence [6-8].

Currently, clinical screening of early ESCA mainly depends on endoscopic observation combined with biopsy-based histopathological diagnosis, however, it can find only $19 \%$ of ESCA at early stages [9]. The reliability of such approaches encounter limitations due to variation in endoscopic sampling site and observer's experience, and may lead to unnecessary biopsies, costs, and high false-positive rates. Even the molecular and cellular changes may appear indolent in endoscopic histology to avoid surveillance $[10,11]$. Thus, it is urgent to explore novel approaches or biomarkers to ameliorate the early detection, treatment guidance, and prognosis prediction for patients. Currently, several 
researchers have reported their exploration of biomarkers for esophageal cancer and their effects [12-14]. Defects of a mitotic checkpoint may bring about mistakes in the chromosome segregation, and the higher level of Cyclin B1 (CCNB1) is a marker of poor prognosis in many cancer types $[15,16]$. However, there are few reports about CCNB1 as a biomarker of ESCA.

Recently, many researchers focus on microarray analysis of gene expression datasets. They succeeded in revealing characteristic genes with the involved key pathways as new cancer biomarkers, with the use of differentially expressed genes(DEGs)and weighted gene co-expression network analysis (WGCNA) [17, 18]. WGCNA tends to identify a co-regulated transcriptional profile of functional gene assemblies, thus enabling a precise network of hub genes and clinical traits [19]. Our study may help understanding the potential molecular mechanism of ESCA initiation and progression and provide a novel prospect for the clinical diagnosis and treatment in ESCA.

\section{Methods}

\section{Data acquisition and preprocessing}

The expression profile of GSE20347, GSE29001, and GSE111044 was achieved based on gene expression omnibus (GEO, https://www.ncbi.nlm.nih.gov/geo/), which is a public database containing comprehensive data of gene profiling and sequencing. GSE20347 included 17 pair-wise ESCA tissues and normal adjacent tissues. GSE29001 included matched normal basal epithelial cells, normal differentiated squamous epithelium, and ESCA from 12 patients. GSE111044 included 3 ESCA tissues and corresponding 3 normal tissues from 3 patients. The experiment GSE20347 and GSE29001 were conducted on platform GPL571 (Affymetrix Human Genome U133A 2.0 Array), and GSE111044 was on platform GPL570 (Affymetrix Human Genome U133 Plus 2.0 Array). After eliminating redundant data (e.g., null value, time), all the gene symbols were matched with probes and were subsequently screened with the 'limma' package of $\mathrm{R}$ software 3.4.1 to perform background correction, quartile normalization, and quantiles summarization.

\section{Identification of DEGs between ESCA and normal tissues}

In this study, we utilized the 'limma' package in bioconductor (http://www.bioconductor.org/) to uncover DEGs between normal and ESCA tissues. Adjusted $P<0.05$ and $\mid \log 2$ fold change $\mid>1$ was set as the criteria for selecting significant DEGs according to the normalized gene expression levels.

\section{Establishment of WGCNA on ESCA}

WGCNA is regard to a methodology to reconstruct a free-scale gene co-expression network and concurrently identify modules consisted of highly correlated genes to appraise connectivity between external clinical traits and the module, in which eigengene is used for summarizing relationship among internal gene membership. In the study, we applied the one-step network construction and module detection function of WGCNA package in R to handle the analysis of microarray dataset GSE70409, which contains 17 cancerous tissues and 17 normal tissues. First, a weighted adjacency matrix was calculated to represent the connecting strength over each pair of gene with outliers removed and ensure a co-expression network. The soft thresholding power was set as 7 to obtain a scale-free topology network. Then, a hierarchical clustering dendrogram was established constituted of abundant branches, and each branch was assigned with a colour to reveal a module. Finally, the modules were used to the association with clinical traits by using module-trait associations according to module membership (MM) and gene significance (GS). Moreover, topological overlap matrix method was utilized for verifying correlation character of eigengenes in different modules.

\section{Module preservation evaluation and principal component analysis (PCA) analysis}

Zsummary is usually used to evaluate the preservation of modules. It takes into consideration several statistics, such as the density and connectivity patterns of module nodes, as well as the overlap among module membership. However, a huge difference in module size is easy to induce deviation in Zsummary value. In our study, the green module had far more genes than the greenyellow module, and consequently, we adopted medianRank, since it eliminates the impact of module size. The module with a lower medianRank has more preservation value than that with a higher medianRank. The finally preserved greenyellow module was processed with PCA to examined the ability of gene in the module to discriminate tumor tissues from normal tissues. PCA was conducted through 'gmodels' and 'scatterplot3d' in R software.

Pathway enrichment analyses of genes in the hub module Gene ontology (GO) is a common method for gene analysis [20]. In the study, we used GO analysis to classify the genes in greenyellow module into three categories based on their bio-function, including biological process (BP), cellular component (CC), and molecular function (MF). Meanwhile, Kyoto encyclopedia of genes and genomes (KEGG) pathway enrichment analysis was 
also performed for exploration of their biological characteristics. Both the GO and KEGG pathway analyses were conducted using the 'clusterProfiler' package in $\mathrm{R}$, with adjusted $P$-value of the analysis calculated by the Benjamini and Hochberg false discovery rate algorithm. Furthermore, a circular chordal graph of the data was generated utilizing gene network analysis via the default euclidean distance and average linkage.

\section{Extract of hub genes from DEGs and the hub module in WGCNA}

39 candidate genes were screened out from the intersection of venn diagram between 3 set of DEGs and the greenyellow module in WGCNA (Fig. 5). CCNB1 was chosen for the next research for the most significant $P$ value. For further study, the protein-protein interaction (PPI) network was constructed among 39 candidate genes (string, https://string-db.org/), and Cytoscape software was applied to visualize the PPI network.

\section{Verification of gene aberrant expression in UALCAN, GEPIA2, and HCMDB}

UALCAN (http://ualcan.path.uab.edu/index.html) is a comprehensive web resource for analyzing cancer OMICS data with the use of TCGA and MET500 databases. In our study, CCNB1 expression data was obtained through 'Expression Analysis' module for the contrast of promoter methylation and gene overall expression level. Further stratified analysis based on gender, age, cancer stages, and histology was also conducted. Additionally, GEPIA2 (http://gepia2.cancer-pku.cn/) and Human Cancer Metastasis Database (HCMDB, http://hcmdb.i-sange r.com/index) analysis was performed to explore the differential expression of CCNB1 between normal tissues and ESCA tissues. GEPIA2 is based on CGA and the GTEx databases with a total of 84 cancer subtypes analysis. HCMDB is designed to examine gene expression of primary and metastasis tumour from 124 previously published transcriptome datasets from TCGA and GEO databases.

Survival analysis with Kaplan-Meier (KM) plotter database KM plotter (http://kmplot.com/) was used to plot the overall survival status and estimate prognostic valve of $C C N B 1$, which is an interactive website containing $54 \mathrm{k}$ genes impact data on survival of 21 cancer types. According to median gene expression level, all patients' survival data was divided into two group: high expression group and low expression group. The $P$ value was set $<0.05$ to ensure statistically significance.
Tumor infiltration analysis of TIMER database

TIMER database provides a systematical analysis of infiltrating abundances in 6 types of immune cells (B cells, CD4+ T cells, CD8+ T cells, neutrophils, macrophages, and dendritic cells) and the infiltration relevant clinical outcome. Hence, we adopted this method to research the tumor infiltration as well as the survival data on CCNB1.

\section{Results}

DEGs screening from normal and cancerous tissues

After preprocessing and normalization (Fig. 1a-c), 1044 DEGs from dataset GSE20347, 1904 from GSE29001, and 2722 from GSE111044 were respectively identified by comparing their expression in cancerous esophageal tissues with normal tissues. As is shown in the volcano plot, there were 571 upregulated and 473 downregulated genes observed in the GSE20347 dataset (Additional file 1: Fig. S1A). Whereas for the dataset GSE29001 and GSE111044, the amount was 894 upregulated genes, 1010 downregulated genes, and 1370 upregulated genes, 1352 downregulated genes, separately (Additional file 1: Fig. S1B, C). The heatmap of upregulated and downregulated genes were shown in Fig. 2a, b.

\section{WGCNA for genes in GSE70409 dataset}

We utilized WGCNA package in $\mathrm{R}$ software to build a weighted co-expression network. The samples of GSE70409 were clustered to filter outlier for subsequent analysis, and one discrete sample (GSM1727139) was noticed and removed out. (Fig. 3a). In our study, the power of $\beta=7$ (scale-free $R 2=0.95$ ) was chosen as the soft-thresholding parameter to ensure a scale-free network (Fig. 3b, c). A total of 32 modules were identified from 19,827 genes, and each module was assigned a colour in hierarchical clustering dendrogram (Fig. 3d). The heatmap was plotted to indicate the similarity of coexpression genes at the network topology level (Fig. 3e). The green module (containing 1906 genes) and greenyellow module (containing 469 genes) were found to have the most prominent module significance (Fig. 3f), and they all shown great clinical meaning (green: correlation coefficient $=-0.92, P<0.01$; greenyellow: correlation coefficient $=0.87, P<0.01$; Fig. $3 g$ ). Clustering of module eigengenes illustrated that the green and greenyellow modules were derived from different metamodules (Fig. 3h). Consequently, the green and greenyellow modules were set as candidate modules for further identification.

\section{Identification of key module and PCA analysis}

In view of the large difference of genes amount in green module than in greenyellow module, we adopted 


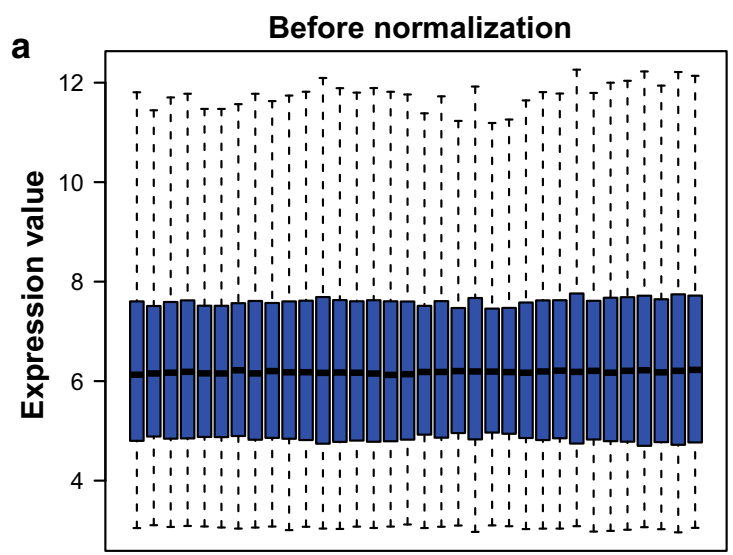

Sample list

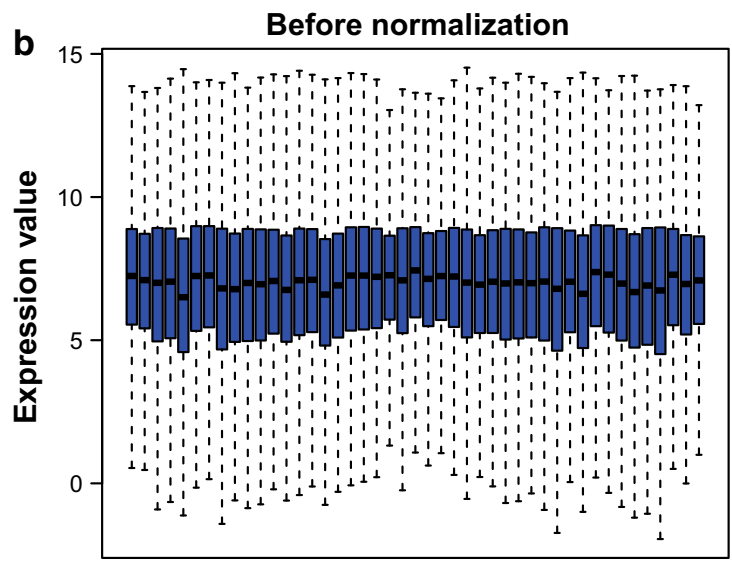

Sample list

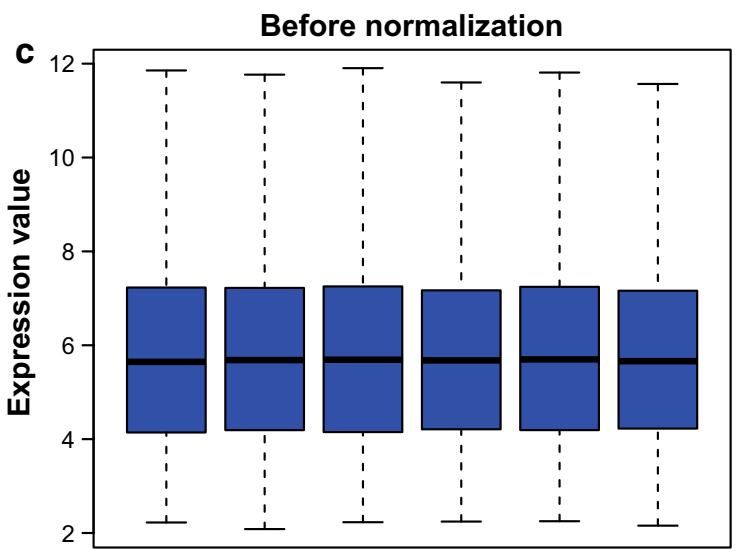

Sample list

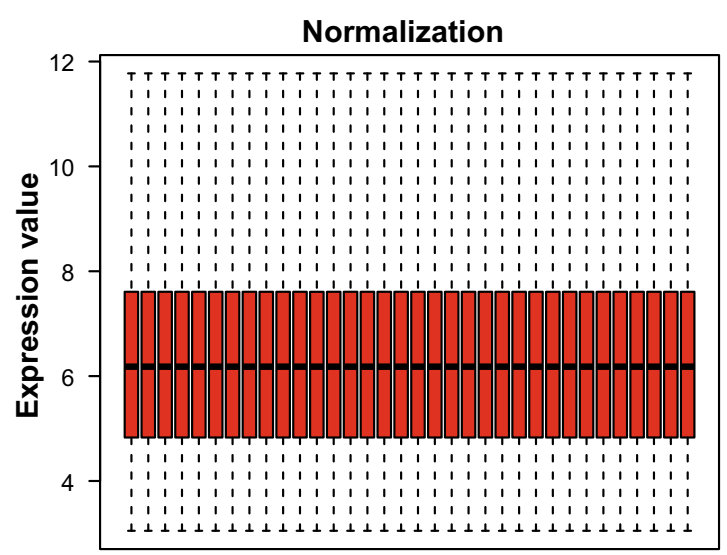

Sample list

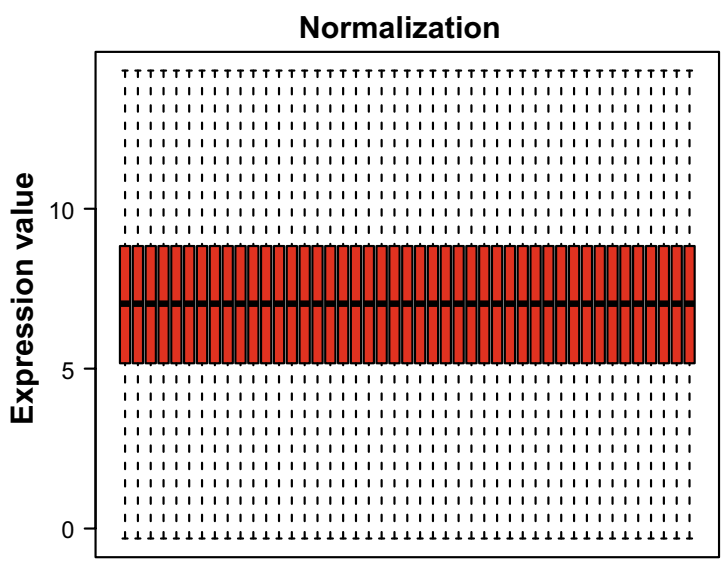

Sample list

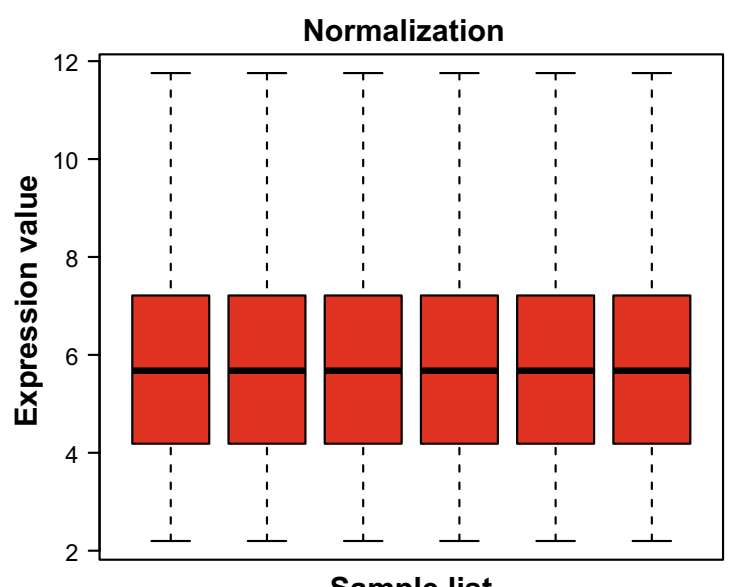

Sample list

Fig. 1 Identification of DEGs. Box plot of genes expression data before and after normalization in GSE20347 (a), GSE29001 (b), and GSE111044 (c)

medianRank with replace of Zsummary to conduct module preservation, because medianRank is more stable in this situation. The result demonstrated that the greenyellow module had a lower medianRank than green module, so it was selected as the key module (Fig. 4a, b). The greenyellow module presented a bunching relationship with purple module in the eigengene adjacency heatmap (Fig. 4c). In addition, the result of PCA indicated a 

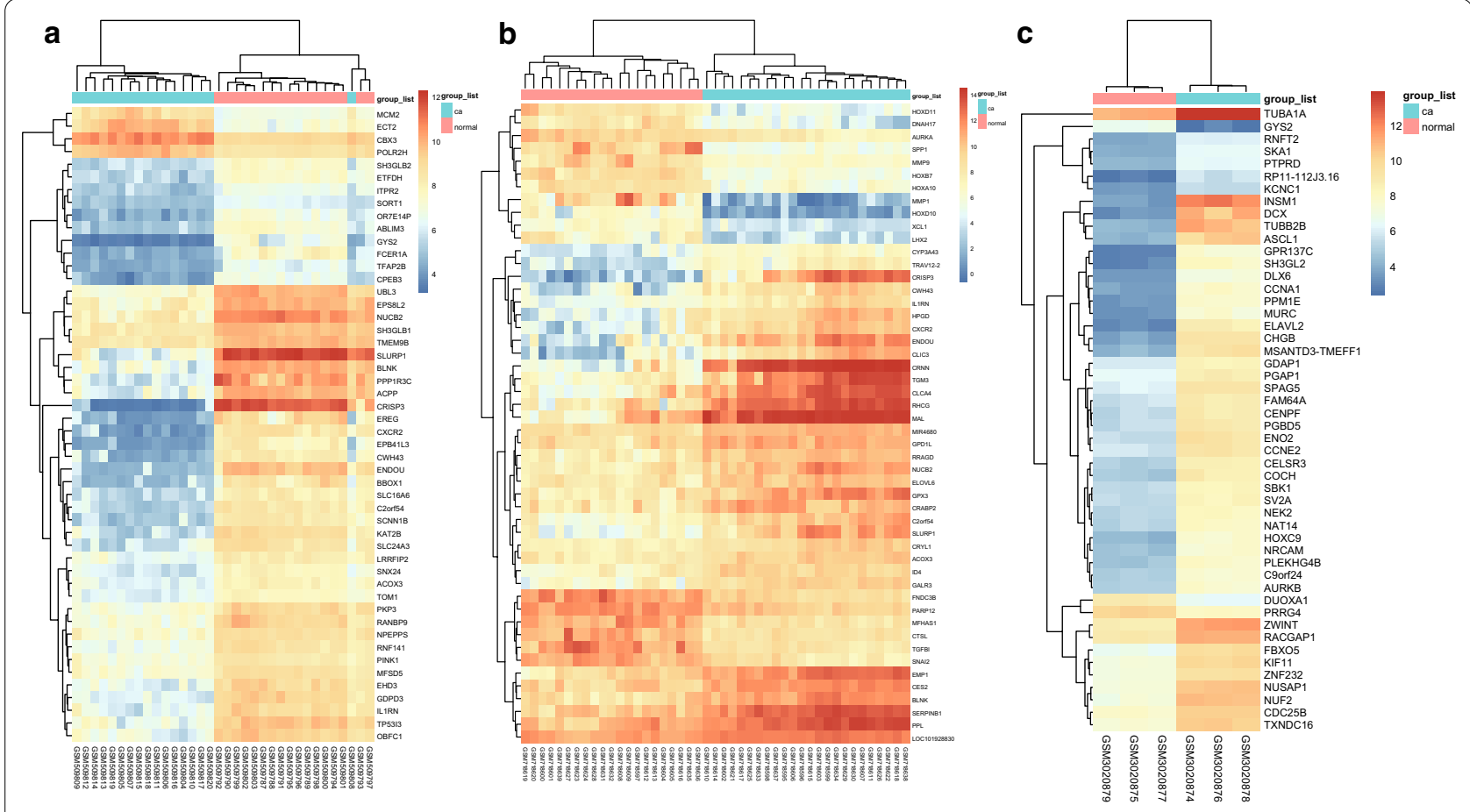

Fig. 2 Heatmap of DEGs. a Heatmap of DEGs in GSE20347. b Heatmap of DEGs in GSE29001. c Heatmap of DEGs in GSE111044

satisfactory concentration of genes within greenyellow module and the great ability to distinguish tumor from normal tissues (Fig. 4d).

\section{GO and KEGG analysis of key module and PPI network construction}

In order to take a deeper insight into the biological function of these genes in greenyellow module, we used the 'clusterProfiler' package in $\mathrm{R}$ to perform the GO and KEGG pathway analyses. The noteworthy pathway of GO analysis is visualized through a chordal graph (Fig. 5a). As presented in Fig. 5c, in BP group, genes were primarily involved in chromosome segregation, mitotic nuclear division, DNA replication, and mitotic sister chromatid segregation; in CC group, genes were markedly enriched in chromosomal region, spindle, chromosome centromeric region, and condensed chromosome; in MF group, the significantly enriched pathway were helicase activity, structural constituent of cytoskeleton, histone kinase activity, and 3'-5' DNA helicase activity. According to
KEGG pathway analysis, the most markedly enriched pathways included oocyte meiosis, cellular senescence, progesterone-mediated oocyte maturation, and cell cycle (Fig. 5d).

Furthermore, the venn diagram showed the intersection 39 genes of DEGs and key module (Additional file 2: Fig. S2), and The CCNB1 was found as hub genes based on the $P$-value. A PPI network was built for those 39 genes (Fig. 5b).

\section{The expression level, survival analysis, and immune infiltration abundance analysis of CCNB1 in patients with ESCA}

The results of GEPIA2, HCMDB, and UALCAN all showed a significantly higher expression level of CCNB1 for patients with ESCA (Fig. 6a-c, $\mathrm{P}<0.01$ ). Promoter methylation level was lower in ESCA patients than in normal people for CCNB1 (Fig. 6d, $\mathrm{P}<0.05$ ). To deeply explore the association between clinicopathological parameters with expression level of CCNB1, stratified

\footnotetext{
(See figure on next page.)

Fig. 3 WGCNA analysis. a Clustering dendrogram of samples with trait heatmap. $\mathbf{b}$ Analysis of the scale-free fit index for various soft-thresholding powers ( $\beta$ ). c Analysis of the mean connectivity for various soft-thresholding powers. $\mathbf{d}$ Cluster dendrogram of genes based on dissimilarity of topological overlap. Each module is assigned a colour, with each branch representing a gene. e Network heatmap based on the selected genes. $\mathbf{f}$ Gene significance distribution of modules. $\mathbf{g}$ Module-trait heatmap of the correlation between module eigengenes and clinical traits of ESCA. $\mathbf{h}$ Cluster dendrogram of eigengenes in modules
} 


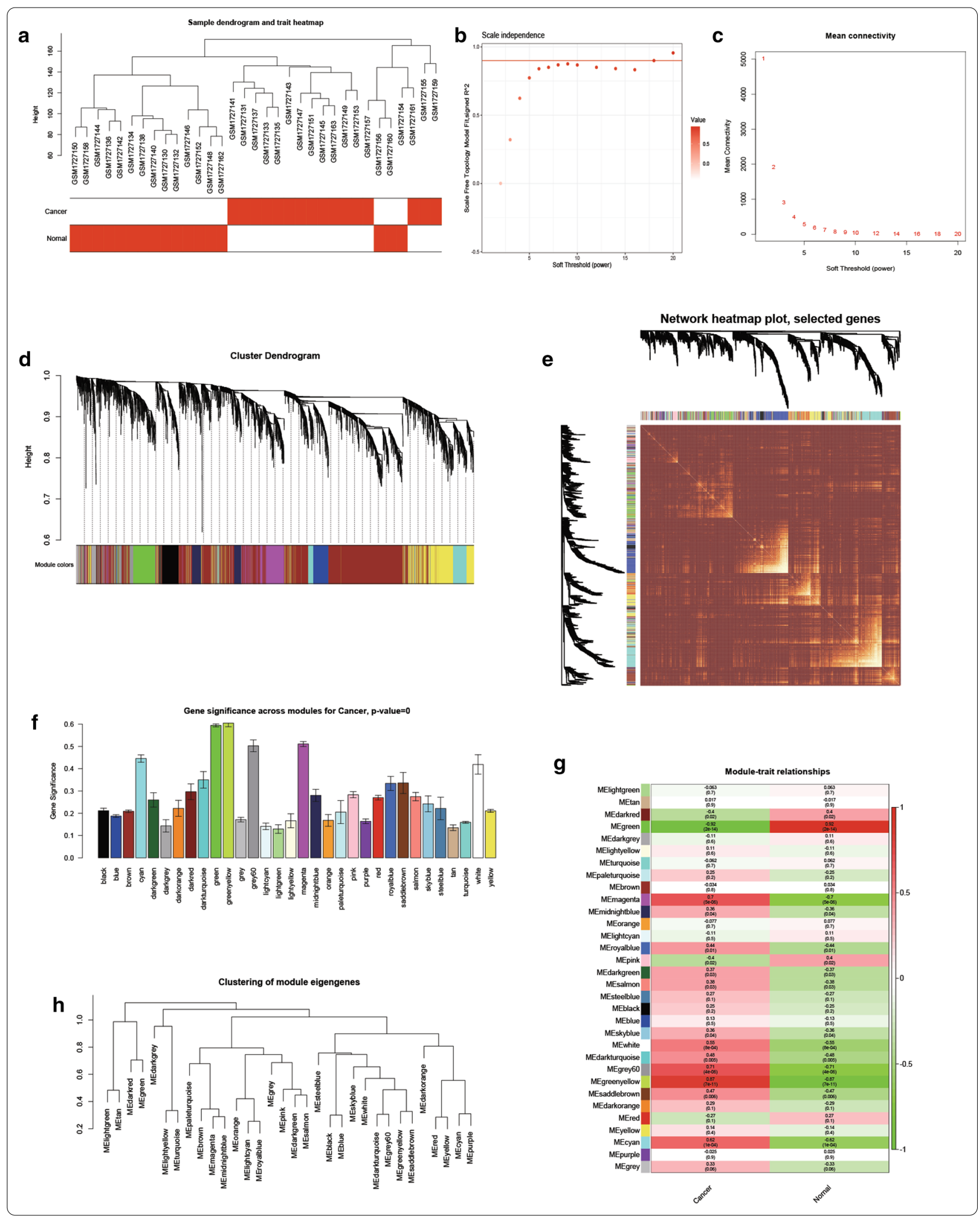




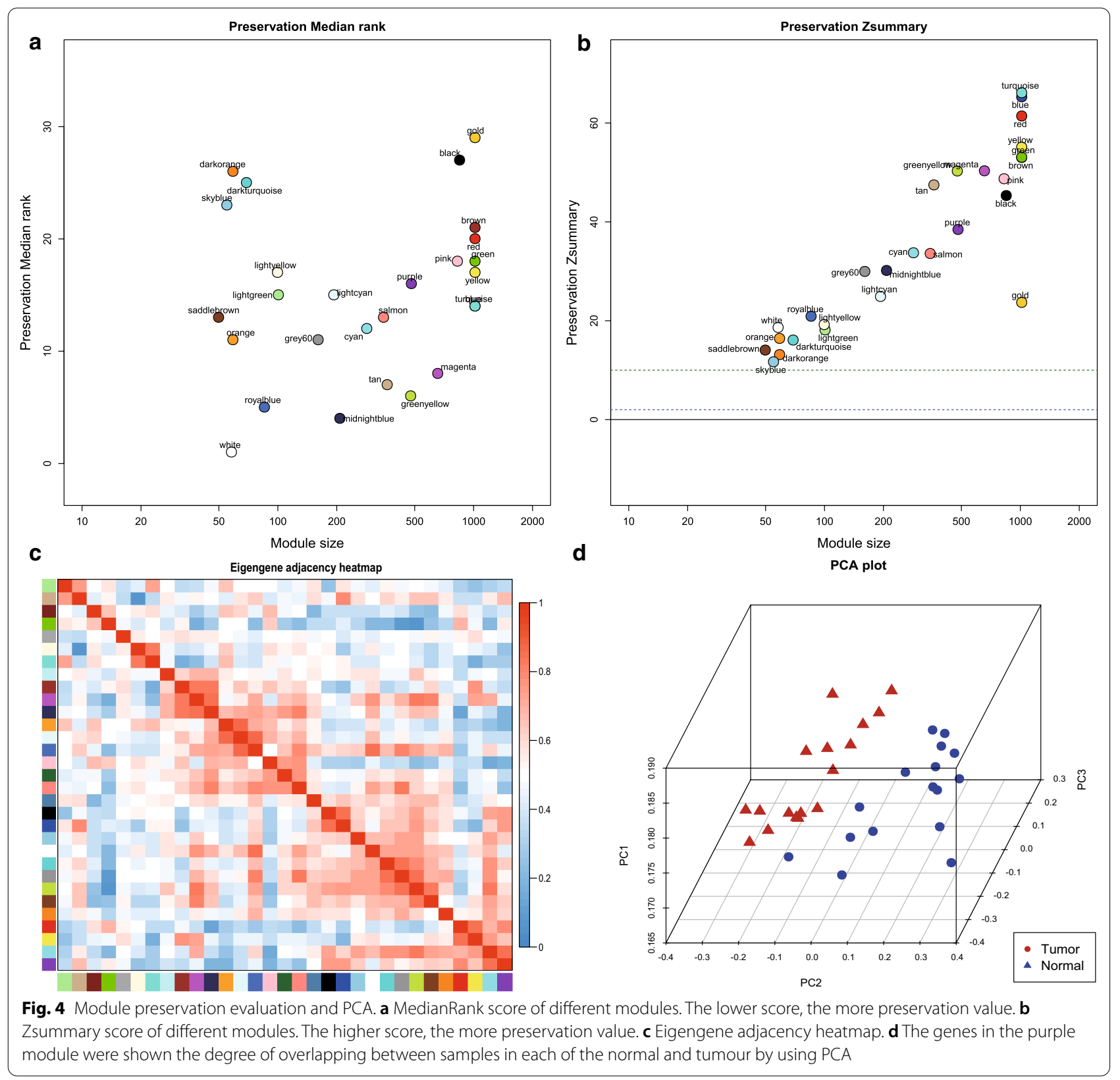

analysis was performed based on patients' gender, age, cancer stages, and histology. As is shown in Fig. 6E, CCNB1 got significantly higher expression in both men and women than in healthy people $(P<0.01)$. People over 40 years old had higher $C C N B 1$ expression (Fig. 6F, $\mathrm{P}<0.01$ ), and the top expression of $C C N B 1$ occurred in 41-60 years. Compared with healthy people, the expression of CCNB1 was higher in ESCA patients of stage 1-4 $(P<0.01)$, and reached the highest expression in stage 2 (Fig. 6g). With regard to histology (Fig. 6h), the expression of CCNB1 in adenocarcinoma or squamous cell carcinoma subtypes was remarkably higher than in normal tissues $(P<0.01)$. Notably, squamous cell carcinoma had more CCNB1 expression than adenocarcinoma, the difference is significant $(P<0.01)$. KM curve demonstrated the better overall survival of ESCA patients in low-risk group than in high-risk group ( $\mathrm{HR}=2.25, P=0.038)$ in Fig. 6i. The correlation among gene expression, immune cells infiltration, and clinical outcome was achieved through TIMER analysis. There was no significant correlation observed between $C C N B 1$ and 6 kinds of immune cells infiltration (Fig. 6j). Furthermore, no significant correlation was observed between infiltration status and cumulative survival (Fig. 6k). 

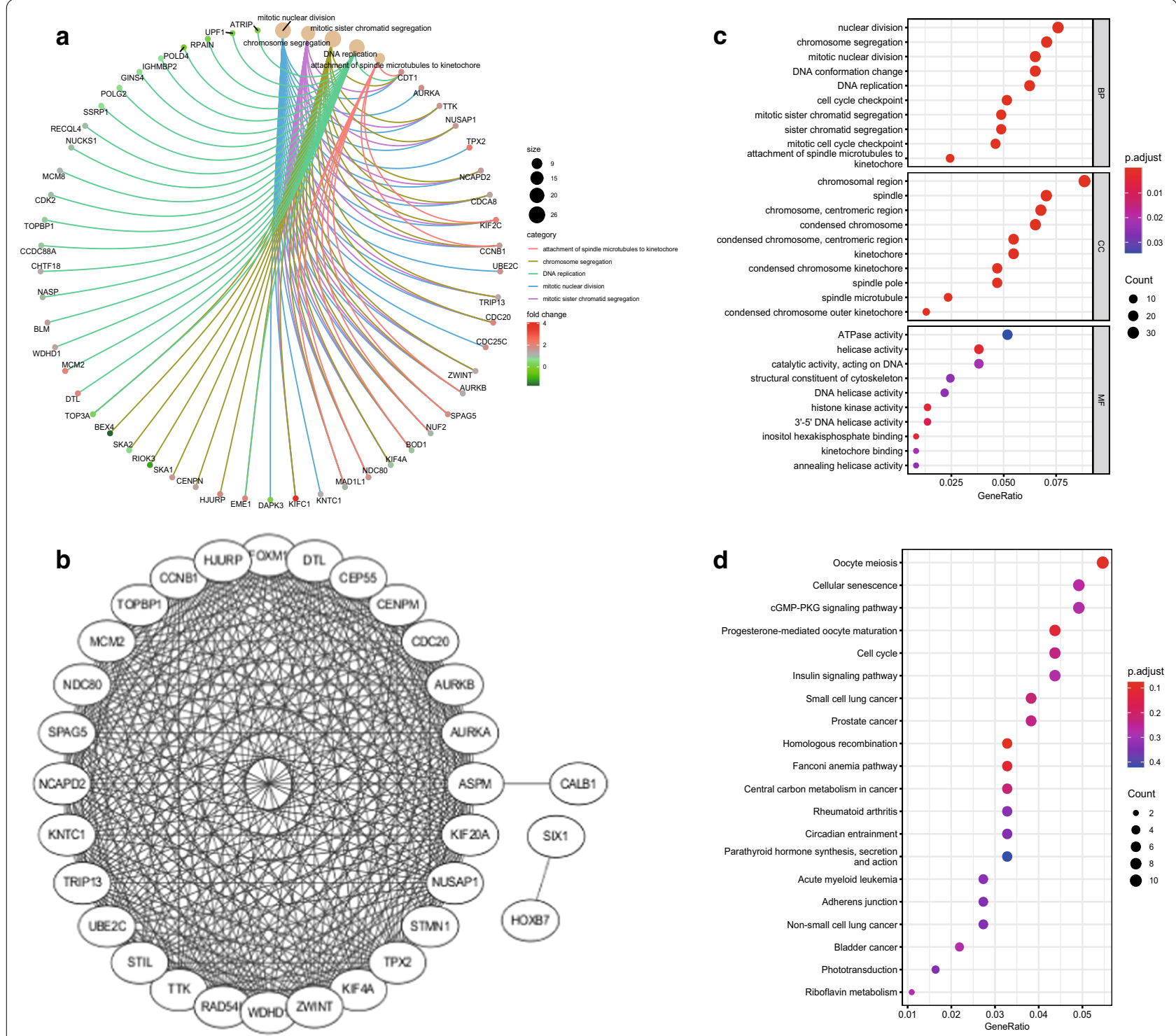

Fig. 5 GO and KEGG enrichment analysis and PPI network. a Chordal graph visualization of part GO analysis. b Protein-protein interaction (PPI) network of 39 joined genes. c GO analysis of genes in key module, through 3 dimensions including biological process (BP), cellular component (CC), and molecular function (MF). d KEGG enrichment analysis of genes in key module

\section{Discussion}

In the present study, we achieved gene microarray data from GEO database and identified DGEs between ESCA patients and normal people. WGCNA was performed to reveal key modules with clinical significance, and greenyellow module was screened out through preservation evaluation. Meanwhile, we applied GO and KEGG analysis to research the mainly

\section{(See figure on next page.)}

Fig. 6 The expression level, survival analysis, and immune infiltration abundance analysis of CCNB1 in patients with ESCA. a HCMDB analysis of aberrant expression of CCNB1 in ESCA patients. b GEPIA2 analysis of aberrant expression of CCNB1 in ESCA patients. c UALCAN analysis of aberrant expression of CCNB1 in ESCA patients. d UALCAN analysis indicated downregulated promoter methylation level of CCNB1 in ESCA patients. $\mathbf{e}-\mathbf{h}$ The different expression level of CCNB1 based on patients' gender, age, stages, and histology were explored in ESCA patients using UALCAN website. $\mathbf{i}$ K-M curve revealing the prognosis valve of CCNB1 in patients with ESCA. $\mathbf{j}$ Correlation analysis between different expressed CCNB1 and immune cell infiltration by utilizing TIMER. $\mathbf{k}$ Prognosis of ESCA patients with different immune infiltration abundance 
a

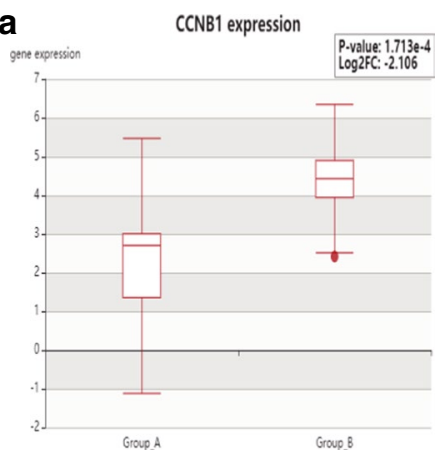

d Promoter methylation level of CCNB1 in ESCA

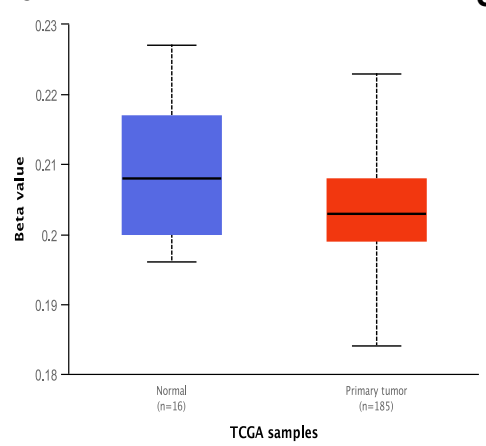

g Expression of CCNB1 in ESCA based on individual cancer
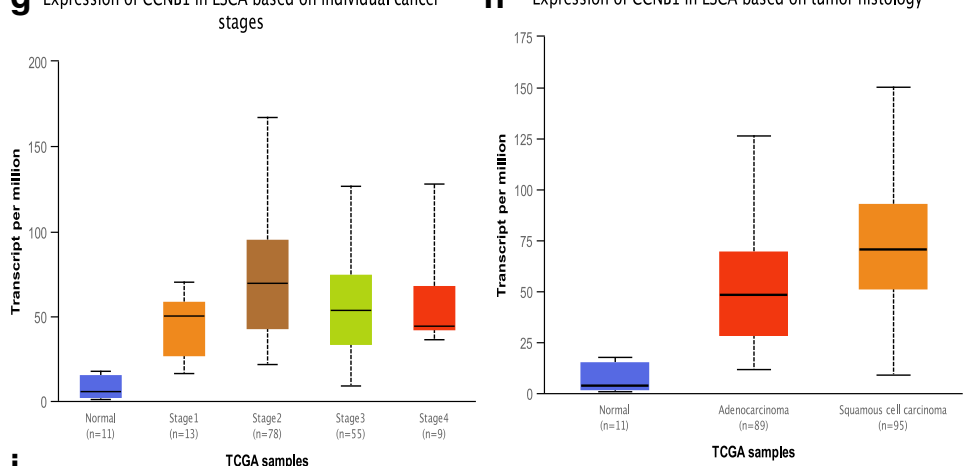

C Expression of CCNB1 in ESCA based on Sample types

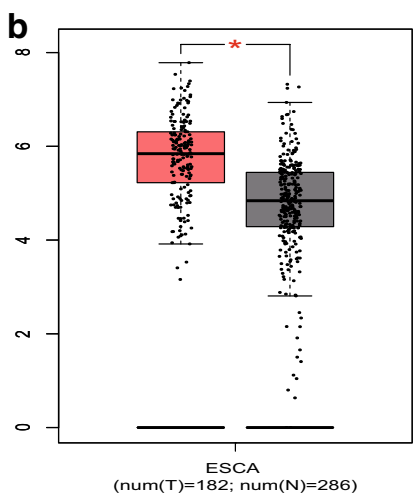

e Expression of CCNB1 in ESCA based on patient's gender
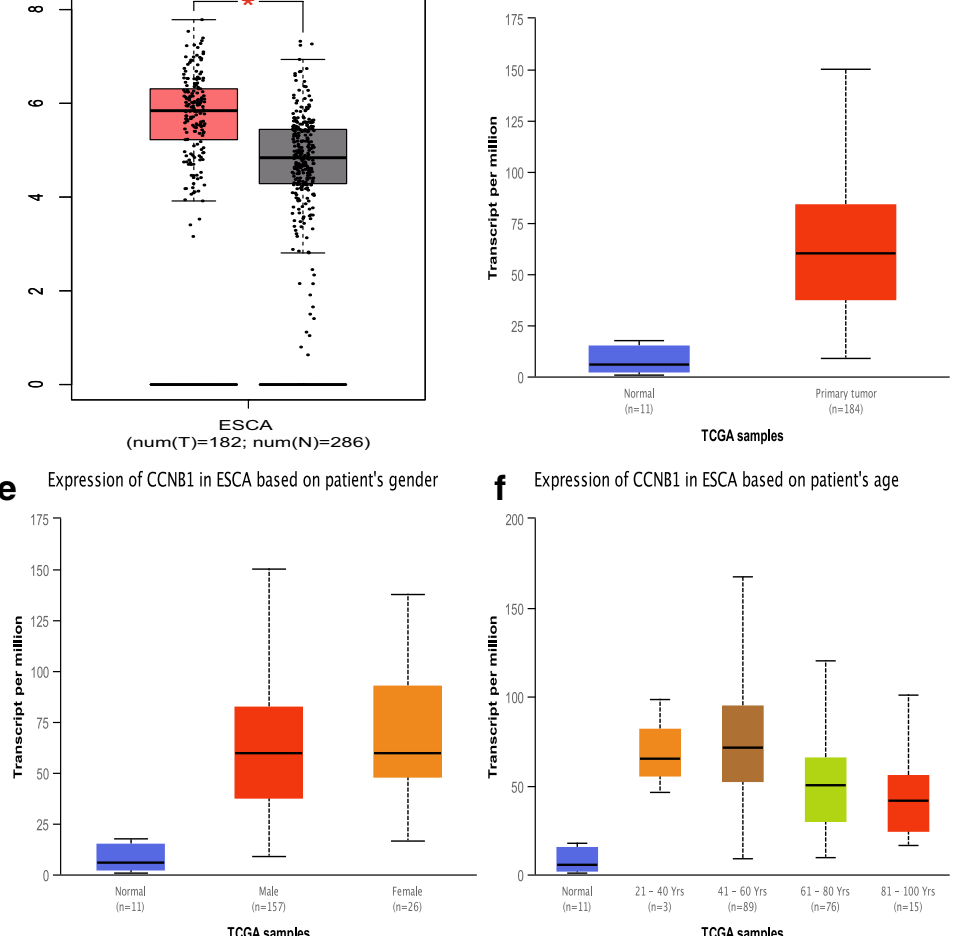

f Expression of CCNB1 in ESCA based on patient's age

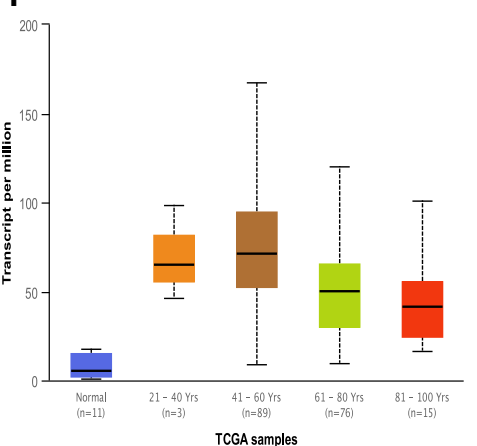

i

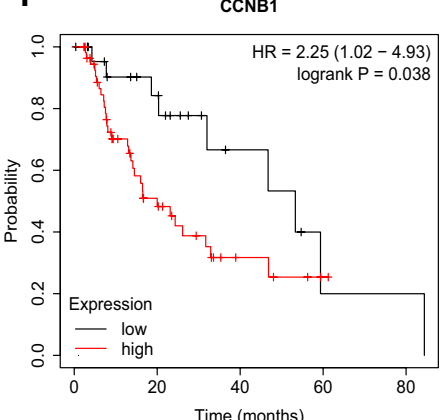

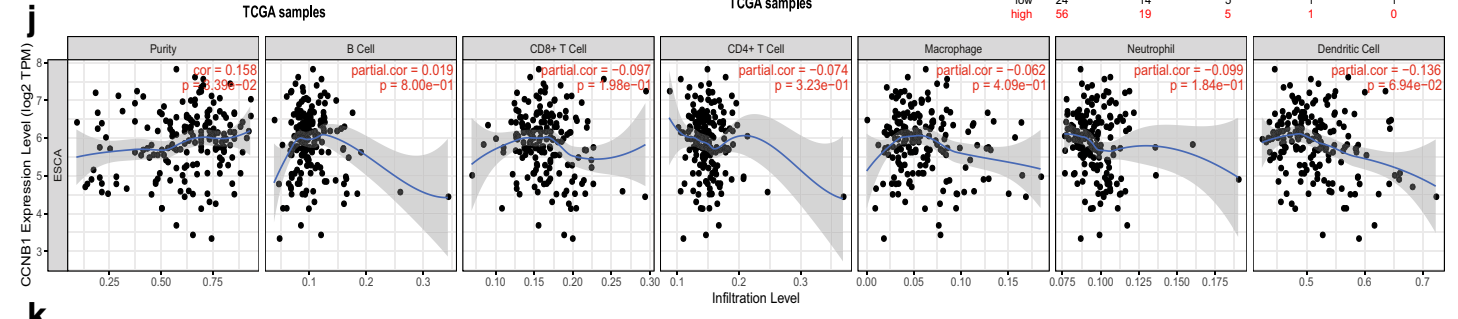

k
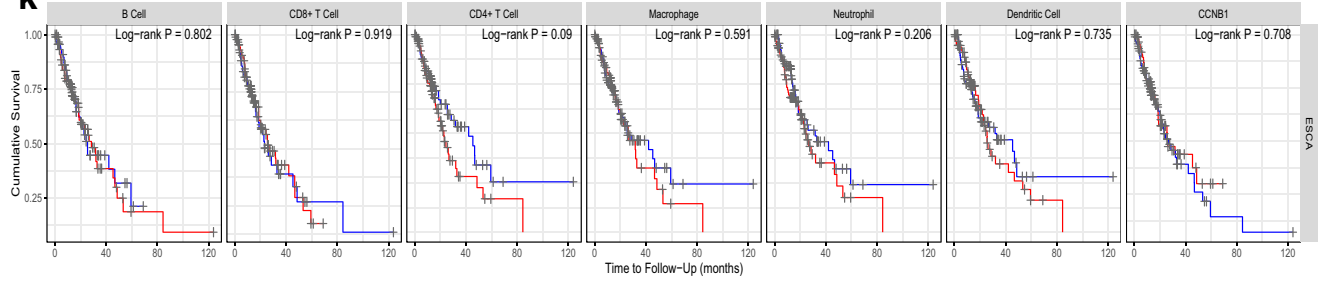

Level 
correlated biological pathway of the hub module. After that, intersection genes were extracted between greenyellow module and DEGs, and a PPI network was built. Finally, CCNB1 was selected and was verified expression and prognostic value through multiple tests.

$\mathrm{GO}$ analysis indicated that the genes in greenyellow module primarily participate in such pathways, including chromosome segregation, mitotic nuclear division, chromosomal region, helicase activity, and structural constituent of cytoskeleton. Some of the pathways have been proved in former studies [21-23]. KEGG pathway analysis found that oocyte meiosis, cellular senescence, progesterone-mediated oocyte maturation, and cell cycle were markedly enriched, and recently Shao et al. also reported important enriched pathways of cellular senescence in ESCC [24, 25].

It is well-known that $C C N B 1$ is involved in cell proliferation by binding to $C D K 1$ to form a complex [26]. The target substrates were phosphorylated by the CCNB1$C D K 1$ complex, which leads to cell cycle progression. Many important steps in mitosis will be initiated by the activated CCNB1-CDK1 complex, including condensation of chromosomes, assembly of spindle pole, and breakdown of nuclear envelope [27, 28], which is consistent with our results. The enrichment analysis suggests that the key module, including CCNB1 in the WGCNA, plays a vital role in mitosis. Furthermore, abnormal CCNB1 expression is often associated with a variety of cancers [29-31], and CCNB1 can be used as a biomarker to predict cancer. However, there have been few reports on the direct correlation between CCNB1 and esophageal cancer, and our research has made important explorations in this area.

Jiang et al. reported that oridonin could downregulate the CCNB1 to arrest the cell cycle in ESCA [32]. Zhang et al. reported that secalonic acid D induced cell G2/M phase arrest by downregulating the expression of CCNB1 and increasing the phosphorylation of $C D K 1$ [33]. Zeng et al. [34] also reported a similar conclusion that EPB41L3 was a potential ESCA suppressor gene and induced $\mathrm{G} 2 / \mathrm{M}$ cell cycle arrest by activating CDK1/CCNB1 signaling. Therefore, these studies all suggested that $C C N B 1$ was correlated with ESCA and clarified the feasibility of a biomarker in ESCA. However, the current studies all focused on mediating the upstream gene of $C C N B 1$ to inhibit the ESCA. Our study focused on the analysis of the role of CCNB1 as a biomarker on ESCA expression and prognosis and achieved good results. In addition, many studies also reported that the sensitivity to chemoradiotherapy for ESCA would increase via arresting the cell cycle at the $\mathrm{G} 2 / \mathrm{M}$ phase and downregulating expression levels of CCNB1 and CDK1 [35-37].
In conclusion, based on DEGs and key modules related to esophageal cancer, CCNB1 was identified as the hub gene, which offered novel insights into the development and treatment of esophageal cancer.

\section{Supplementary Information}

The online version contains supplementary material available at https://doi. org/10.1186/s12935-021-01826-x.

Additional file 1: Figure S1. Volcano plot of up-regulated and downregulated DEGs in GSE20347(A), GSE29001(B), and GSE111044(C).

Additional file 2: Figure S2. Venn diagram. Venn diagram indicated overlapping 39 hub genes of the DEG and WGCNA.

\section{Abbreviations}

DEGs: Differential expression genes; WGCNA: Weighted gene co-expression network analysis; ESCA: Esophageal cancer; ESCC: Esophageal squamous cell carcinoma; CCNB1: Cyclin B1; MM: Module membership; GS: Gene significance; PCA: Principal component analysis; GO: Gene Ontology; BP: Biological process; CC: Cellular component; MF: Molecular function; KEGG: Kyoto encyclopedia of genes and genomes; PPI: Protein-protein interaction; HCMDB: Human cancer metastasis database; KM: Kaplan-Meier.

\section{Acknowledgements}

Not applicable.

\section{Authors' contributions}

RW contributed to the conception and revised the work critically for important intellectual content and approved the version to be published and agreed to be accountable for all aspects of the work in ensuring that questions related to the accuracy and integrity of any part of the work were appropriately investigated and resolved. HZ, Y-KM, and J-YS contributed to the design of the work and drafted the work and approved the version to be published and agreed to be accountable for all aspects of the work in ensuring that questions related to the accuracy. L-LZ, LL, and Z-NF contributed to the conception and revised the work critically for important intellectual content and approved the version to be published and agreed to be accountable for all aspects of the work in ensuring that questions related to the accuracy and integrity of any part of the work were appropriately investigated and resolved. All authors read and approved the final manuscript.

\section{Funding}

Jiangsu Province"333" project (Award Number: BRA2018388, Grant Recipient: Li Liu). Six Talent Peaks Project in Jiangsu Province (Award Number:WSW-006, Grant Recipient: Li Liu).

\section{Availability of data and materials}

The datasets used and/or analyzed during the present study are available from the corresponding author on reasonable request.

Ethics approval and consent to participate Not applicable.

Patient consent for publication

Not applicable.

Competing interests

The authors declare that they have no competing interests.

\section{Author details}

${ }^{1}$ Department of Digestive Endoscopy, The First Affiliated Hospital with Nanjing Medical University, 300 Guangzhou Road, Nanjing 210029, Jiangsu, China. ${ }^{2}$ Nanjing Medical University, 101 Longmian Avenue, Nanjing, Jiangsu, China.

${ }^{3}$ Department of Cardiology, The First Affiliated Hospital with Nanjing Medical University, 300 Guangzhou Road, Nanjing 210029, Jiangsu, China. 
Received: 21 November 2020 Accepted: 9 February 2021

Published online: 25 February 2021

\section{References}

1. Bray F, Ferlay J, Soerjomataram I, Siegel RL, Torre LA, Jemal A. Global cancer statistics 2018: GLOBOCAN estimates of incidence and mortality worldwide for 36 cancers in 185 countries. CA Cancer J Clin. 2018;68(6):394-424.

2. Wei WQ, Hao CQ, Guan CT, Song GH, Wang M, Zhao DL, Li BY, Bai WL, Hou PY, Wang JW, et al. Esophageal histological precursor lesions and subsequent 8.5-year cancer risk in a population-based prospective study in China. Am J Gastroenterol. 2020;115(7):1036-44.

3. He LJ, Xie C, Wang ZX, Li Y, Xiao YT, Gao XY, Shan HB, Luo LN, Chen LM, Luo GY, et al. Submucosal saline injection followed by endoscopic ultrasound versus endoscopic ultrasound only for distinguishing between T1a and T1b esophageal cancer. Clin Cancer Res. 2020;26(2):384-90.

4. Martin JL, Wolanin A, Lerner I. Oral cancer screening. reducing fear using salivary diagnostics. Dent Today. 2016;35(5):12.

5. Khan T, Relitti N, Brindisi M, Magnano S, Zisterer D, Gemma S, Butini $\mathrm{S}$, Campiani G. Autophagy modulators for the treatment of oral and esophageal squamous cell carcinomas. Med Res Rev. 2020;40(3):1002-60.

6. Azad TD, Chaudhuri AA, Fang P, Qiao Y, Esfahani MS, Chabon JJ, Hamilton EG, Yang YD, Lovejoy A, Newman AM, et al. Circulating tumor DNA analysis for detection of minimal residual disease after chemoradiotherapy for localized esophageal cancer. Gastroenterology. 2020;158(3):494-505.

7. Mariette C, Markar S, Dabakuyo-Yonli TS, Meunier B, Pezet D, Collet D, D'Journo XB, Brigand C, Perniceni T, Carrere N, et al. Health-related quality of life following hybrid minimally invasive versus open esophagectomy for patients with esophageal cancer, analysis of a multicenter, openlabel, randomized phase III controlled trial: the MIRO trial. Ann Surg. 2020;271(6):1023-9.

8. Berkelmans G, Fransen L, Dolmans-Zwartjes A, Kouwenhoven EA, van Det MJ, Nilsson M, Nieuwenhuijzen G, Luyer M. Direct oral feeding following minimally invasive esophagectomy (NUTRIENT II trial): an international, multicenter, open-label randomized controlled trial. Ann Surg. 2020;271(1):41-7

9. Kossatz S, Pirovano G, Demetrio DSFP, Strome AL, Sunny SP, Zanoni DK, Mauguen A, Carney B, Brand C, Shah V, et al. Validation of the use of a fluorescent PARP1 inhibitor for the detection of oral, oropharyngeal and oesophageal epithelial cancers. Nat Biomed Eng. 2020;4(3):272-85.

10. Davison JM, Goldblum J, Grewal US, McGrath K, Fasanella K, Deitrick C, DeWard AD, Bossart EA, Hayward SL, Zhang Y, et al. Independent blinded validation of a tissue systems pathology test to predict progression in patients with Barrett's esophagus. Am J Gastroenterol. 2020;115(6):843-52.

11. Tan MC, Bhushan S, Quang T, Schwarz R, Patel KH, Yu X, Li Z, Wang G, Zhang F, Wang $X$, et al. Automated software-assisted diagnosis of esophageal squamous cell neoplasia using high-resolution microendoscopy. Gastrointest Endosc. 2020. https://doi.org/10.1016/j.gie.2020.07.007.

12. Rehman AU, Iqbal MA, Sattar R, Saikia S, Kashif M, Ali WM, Medhi S, Saluja SS, Husain SA. Elevated expression of RUNX3 co-expressing with EZH2 in esophageal cancer patients from India. Cancer Cell Int. 2020;20:445.

13. Hesari A, Azizian M, Sheikhi A, Nesaei A, Sanaei S, Mahinparvar N, Derakhshani M, Hedayt P, Ghasemi F, Mirzaei H. Chemopreventive and therapeutic potential of curcumin in esophageal cancer: current and future status. Int J Cancer. 2019;144(6):1215-26.

14. Jamali L, Tofigh R, Tutunchi S, Panahi G, Borhani F, Akhavan S, Nourmohammadi P, Ghaderian S, Rasouli M, Mirzaei H. Circulating microRNAs as diagnostic and therapeutic biomarkers in gastric and esophageal cancers. J Cell Physiol. 2018;233(11):8538-50.

15. Wang S, Sun H, Zhan X, Wang Q. MicroRNA718 serves a tumorsuppressive role in nonsmall cell lung cancer by directly targeting CCNB1. Int J Mol Med. 2020;45(1):33-44.

16. Bai X, Wang W, Zhao P, Wen J, Guo X, Shen T, Shen J, Yang X. LncRNA CRNDE acts as an oncogene in cervical cancer through sponging miR183 to regulate CCNB1 expression. Carcinogenesis. 2020:41(1):111-21.

17. Nangraj AS, Selvaraj G, Kaliamurthi S, Kaushik AC, Cho WC, Wei DQ. Integrated PPI- and WGCNA-retrieval of hub gene signatures shared between
Barrett's esophagus and esophageal adenocarcinoma. Front Pharmacol. 2020;11:881.

18. Xiong Y, Yuan L, Xiong J, Xu H, Luo Y, Wang G, Ju L, Xiao Y, Wang X. An outcome model for human bladder cancer: a comprehensive study based on weighted gene co-expression network analysis. J Cell Mol Med. 2020;24(3):2342-55

19. Radulescu E, Jaffe AE, Straub RE, Chen Q, Shin JH, Hyde TM, Kleinman JE, Weinberger DR. Identification and prioritization of gene sets associated with schizophrenia risk by co-expression network analysis in human brain. Mol Psychiatry. 2020;25(4):791-804.

20. Harris MA, Clark J, Ireland A, Lomax J, Ashburner M, Foulger R, Eilbeck K, Lewis S, Marshall B, Mungall C, et al. The Gene Ontology (GO) database and informatics resource. Nucleic Acids Res. 2004;32(Database issue):D258-61.

21. Li B, Li YM, He WT, Chen H, Zhu HW, Liu T, Zhang JH, Song TN, Zhou YL. Knockdown of DDX46 inhibits proliferation and induces apoptosis in esophageal squamous cell carcinoma cells. Oncol Rep. 2016;36(1):223-30.

22. Zhan XH, Jiao JW, Zhang HF, Xu XE, He JZ, Li RL, Zou HY, Wu ZY, Wang $\mathrm{SH}$, Wu JY, et al. LOXL2 upregulates phosphorylation of ezrin to promote cytoskeletal reorganization and tumor cell invasion. Cancer Res. 2019;79(19):4951-64.

23. Krause L, Nones K, Loffler KA, Nancarrow D, Oey H, Tang YH, Wayte NJ, Patch AM, Patel K, Brosda S, et al. Identification of the CIMP-like subtype and aberrant methylation of members of the chromosomal segregation and spindle assembly pathways in esophageal adenocarcinoma. Carcinogenesis. 2016;37(4):356-65.

24. Shao M, Li W, Wang S, Liu Z. Identification of key genes and pathways associated with esophageal squamous cell carcinoma development based on weighted gene correlation network analysis. J Cancer. 2020;11(6):1393-402.

25. Pourhanifeh MH, Vosough M, Mahjoubin-Tehran M, Hashemipour M, Nejati M, Abbasi-Kolli M, Sahebkar A, Mirzaei H. Autophagy-related microRNAs: possible regulatory roles and therapeutic potential in and gastrointestinal cancers. Pharmacol Res. 2020;161:105133.

26. Hayward D, Alfonso-Perez T, Gruneberg U. Orchestration of the spindle assembly checkpoint by CDK1-cyclin B1. FEBS Lett. 2019:593(20):2889-907.

27. Fang L, Du WW, Awan FM, Dong J, Yang BB. The circular RNA circ-Ccnb1 dissociates Ccnb1/Cdk1 complex suppressing cell invasion and tumorigenesis. Cancer Lett. 2019;459:216-26

28. Yuan J, Kramer A, Matthess Y, Yan R, Spankuch B, Gatje R, Knecht R, Kaufmann M, Strebhardt K. Stable gene silencing of cyclin B1 in tumor cells increases susceptibility to taxol and leads to growth arrest in vivo. Oncogene. 2006;25(12):1753-62.

29. Zhang H, Zhang X, Li X, Meng WB, Bai ZT, Rui SZ, Wang ZF, Zhou WC, Jin $X D$. Effect of CCNB1 silencing on cell cycle, senescence, and apoptosis through the p53 signaling pathway in pancreatic cancer. J Cell Physiol. 2018:234(1):619-31.

30. Fang L, Du WW, Lyu J, Dong J, Zhang C, Yang W, He A, Kwok Y, Ma J, Wu N, et al. Enhanced breast cancer progression by mutant p53 is inhibited by the circular RNA circ-Ccnb1. Cell Death Differ. 2018;25(12):2195-208.

31. Zhuang L, Yang Z, Meng Z. Upregulation of BUB1B, CCNB1, CDC7, CDC20, and MCM3 in tumor tissues predicted worse overall survival and disease-free survival in hepatocellular carcinoma patients. Biomed Res Int. 2018:2018:7897346.

32. Jiang JH, Pi J, Jin H, Cai JY. Oridonin-induced mitochondria-dependent apoptosis in esophageal cancer cells by inhibiting PI3K/AKT/mTOR and Ras/Raf pathways. J Cell Biochem. 2019;120(3):3736-46.

33. Zhang H, Huang L, Tao L, Zhang J, Wang F, Zhang X, Fu L. Secalonic acid $D$ induces cell apoptosis in both sensitive and ABCG2-overexpressing multidrug resistant cancer cells through upregulating c-Jun expression. Acta Pharm Sin B. 2019;9(3):516-25.

34. Zeng R, Liu Y, Jiang ZJ, Huang JP, Wang Y, Li XF, Xiong WB, Wu XC, Zhang JR, Wang QE, et al. EPB41L3 is a potential tumor suppressor gene and prognostic indicator in esophageal squamous cell carcinoma. Int J Oncol. 2018;52(5):1443-54

35. Zheng R, Liu Y, Zhang X, Zhao P, Deng Q. miRNA-200c enhances radiosensitivity of esophageal cancer by cell cycle arrest and targeting P21. Biomed Pharmacother. 2017:90:517-23. 
36. Ma Y, Wang B, Guo Y, Zhang Y, Huang S, Bao X, Bai M. Inhibition of miR$196 a$ affects esophageal cancer cell growth in vitro. Biomed Pharmacother. 2016;84:22-7.

37. Huang L, Wang Y, Chen J, Wang Y, Zhao Y, Wang Y, Ma Y, Chen X, Liu W, $\mathrm{Li} Z$, et al. Long noncoding RNA PCAT1, a novel serum-based biomarker, enhances cell growth by sponging miR-326\&nbsp;in oesophageal squamous cell carcinoma. Cell Death Dis. 2019;10(7):513.

\section{Publisher's note}

Springer Nature remains neutral with regard to jurisdictional claims in published maps and institutional affiliations.
Ready to submit your research? Choose BMC and benefit from:

- fast, convenient online submission

- thorough peer review by experienced researchers in your field

- rapid publication on acceptance

- support for research data, including large and complex data types

- gold Open Access which fosters wider collaboration and increased citations

- maximum visibility for your research: over 100M website views per year

At BMC, research is always in progress.

Learn more biomedcentral.com/submissions 